\title{
¿EXISTEN INDICADORES PARA IDENTIFICAR EL TALENTO?
}

\section{Are there indicators for identifying talent?}

M. Teresa González Astudillo y Fernando Sergio Domingues Carlos

Universidad de Salamanca. Departamento de Didáctica de la Matemática y las Ciencias Experimentales

Correo-e: maite@usal.es; sergiodcarlos@gmail.com

Recepción: 20 de noviembre de 2014

Envío a informantes: 5 de diciembre de 2014

Aceptación definitiva: 28 de enero de 2015

Biblid. [02I4-3402 (20I5) (II época) n. ${ }^{\circ}$ 2I; 2I-32]

Resumen: A lo largo de las últimas décadas los investigadores han aportado diferentes aproximaciones de la noción de talento desde diferentes perspectivas diferenciándolo de otros conceptos como superdotado, experto, genio u otros similares. En estas páginas intentaremos describir de forma sucinta las diferentes definiciones que han surgido durante estos últimos años así como dar una muestra de algunos de los indicadores que se considera que caracterizan a los sujetos que poseen algún talento.

Palabras Clave: talento; educación; indicadores.

АвsтRACT: Researchers have been made different approaches over the past decades, to the notion of talent from different perspectives differentiating this concept from others such as gifted, expert, genius or similar ones. Along these pages we attempt to describe succinctly different definitions that have emerged in recent years as well as giving a sample of some of the indicators that characterize the subjects that have some talent.

KEY WORDS: talent; education; indicators.

\section{Introducción}

$\mathrm{L}$

A EDUCACIÓN PARA TODOS ES UNA MÁXIMA DEL MUNDO EDUCATIVO ACTUAL, pero podríamos preguntarnos hasta qué punto esto se lleva a la práctica en todos los casos, como en lo que se refiere a los alumnos con talento o con altas capacidades. ¿Reciben estos alumnos una formación adecuada para desarrollarse personalmente, 
socialmente e intelectualmente? ¿Están los maestros y profesores formados e informados para proporcionar a estos alumnos las experiencias educativas adecuadas a sus capacidades? Estas son algunas de las preguntas que podemos realizar y que no tienen una fácil respuesta. Bien es cierto que los últimos años se ha hecho un gran esfuerzo por establecer baremos y pruebas de detección de los alumnos con altas capacidades e incluso de dotarles de una atención específica, aunque fuera del horario escolar.

Este monográfico está dedicado a poner de relieve un problema que se produce en las aulas y al que se ha dado poca atención aunque, como veremos en cada uno de los artículos que lo componen, se está progresando desde el punto de vista de la investigación y cada vez hay más especialistas entregados a este tema de estudio.

Desde el punto de vista de la educación hay una gran disparidad en diferentes aspectos relativos a esta temática. Una de las cuestiones en las que surge la controversia es en relación con los términos usados para designar a los sujetos de la investigación: superdotados, alumnos con talento, altas capacidades, alto rendimiento, prodigio, genio, excelencia, experto... Otro elemento de variedad es en relación con la noción de inteligencia, así mientras que para Spearman es única, para otros como Gardner habría que hablar de inteligencias múltiples. Tampoco está claro, en cuanto a la detección del talento, el límite o línea que determina que una persona se considere o no superdotada. Además se han identificado diferentes aspectos que condicionan el talento como la creatividad, la motivación o el pensamiento divergente, pero no está claro que todos ellos lo constituyan o en qué medida la posesión de cada uno de ellos es un indicativo del talento.

\section{2. ¿Qué es el talento?}

Superdotado, talento, genio son términos que se utilizan para hablar de las personas que tienen habilidades fuera de la norma, pero ¿existen matices diferenciadores entre ellos? Intentemos asociar una definición a cada uno de estos términos con el fin de aclarar confusiones.

Cuando se habla de una persona con altas capacidades se hace referencia esencialmente a la habilidad intelectual, creatividad y motivación que se presentan en un grado por encima de la media. El término Altas capacidades intelectuales es un término regulado como tal en la Ley Orgánica 2/2006, de 3 de mayo, de Educación, especificado en los artículos 76 y 77 de la misma. En la normativa legal se recoge esta terminología para favorecer una concepción más abierta del alumnado excepcionalmente dotado y que su potencial intelectual no está sometido a un momento concreto de su desarrollo (Martínez y otros, 20I2).

La Organización Mundial de la Salud (oms) define a una persona superdotada como aquella que cuenta con un cociente intelectual superior a I30. Esto constituye un perfil complejo, donde todos los recursos intelectuales aparecen en un nivel elevado y se refiere a la dotación, potencial o actual, que caracteriza de forma sobresaliente y prioritaria a ciertos individuos de ambos sexos, generalmente procedente de la capacidad intelectual (Prieto y Castejón, 2000).

La persona que presenta talento puede definirse como aquella que muestra una elevada aptitud en un ámbito o tipo de información (por ejemplo, el talento matemático). Así mismo responde a la especialidad y las diferencias cuantitativas altas sobre 
todo en velocidad de ejecución y automatización de un proceso, siendo el resto de procesamientos generalmente dentro de la media o en ocasiones un tanto discretos.

Podemos definir el talento como una elevada aptitud, tipología de procesamiento o habilidad excepcional en una, dos o más áreas del conocimiento. En las áreas en las que posee algún talento es posible que la persona con frecuencia sea más efectiva que, por ejemplo, un superdotado, sin embargo, en el resto de ámbitos o de formas de procesamiento se manifiestan dentro de la media o incluso de forma deficitaria.

Castelló y Battle (1998) clasifican los diferentes talentos en dos grupos:

- Talentos simples o múltiples: matemático, lógico, social, creativo y verbal.

- Talentos complejos: académico y artístico-figurativo.

En la escuela los profesores suelen detectar fácilmente los talentos académicos en los que se combina el talento lógico con el verbal y la gestión académica. Los alumnos que poseen este tipo de talento rinden mucho desde el punto de vista académico y son capaces de absorber gran cantidad de información.

Los alumnos precoces son aquellos que se desarrollan a una edad más temprana, suelen madurar antes y pueden adquirir conocimientos a edades más tempranas o manifestar destrezas antes de lo normal.

Por otro lado, se considera experto a una persona que por acumulación de conocimientos manifiesta una amplia competencia en uno o varios dominios (Chi, 2006) aunque también se puede definir en sentido relativo indicando que un perito es aquel que se distingue por su actuación respecto del resto de las personas (Cianciolo, Matthew, Sternberg y Wagner, 2006).

\section{Modelos sobre el talento}

Especialmente interesante para los filósofos e investigadores es la cuestión de hasta qué punto el talento se basa en dotes innatas o es el resultado de la adquisición de conocimientos y procede de la experiencia. Así, mientras que algunos investigadores consideran la excelencia como un resultado de las habilidades innatas de los individuos, aunque incluyen la contribución de algunas características como la motivación y la personalidad (por ejemplo, Renzulli, 1978), para otros, el papel de la dotación genética no es determinante, pues, aunque se reconoce su importancia, consideran que los altos niveles de rendimiento se explican mejor por la práctica deliberada prolongada (Ericsson, 2009; VanLehn y Van De Sande, 2009; Zimmerman, 2006). Así, observamos la existencia de un continuo de perspectivas teóricas, desde aquellas que ponen el énfasis en la dotación genética, y sugieren hablar más adecuadamente de superdotados, hasta aquellas que privilegian la importancia de la práctica continua, generalmente considerando el concepto de excelencia. Echemos un vistazo a algunos de estos enfoques teóricos, empezando por el primer grupo y acercándonos progresivamente al segundo teniendo en cuenta que en todos ellos subyace como idea común la noción de inteligencia.

a) Modelo de los tres anillos de Renzulli

Uno de los modelos es de los tres anillos de Renzulli para el que el talento no es un constructo unidimensional, sino que se basa en la combinación de tres características: 
- Habilidad general por encima de la media.

- Alto nivel de compromiso con la tarea.

- Alto nivel de creatividad.

Las habilidades superiores a la media, de acuerdo con este diseño, pueden ser de aplicación general (como la capacidad de procesar información para integrar los resultados de experimentos anteriores con el fin de responder mejor a nuevas situaciones o la capacidad de razonar de manera abstracta) o específica a un dominio particular (como la capacidad de adquirir conocimiento o habilidad en un contexto específico, por ejemplo, en matemáticas). El segundo anillo, llamado por Renzulli compromiso con la tarea, se asocia con características (por ejemplo, la persistencia, la voluntad de trabajar insistentemente o por cuenta propia) relacionadas con la realización de una tarea concreta. A su vez, la creatividad está asociada a la capacidad de un individuo para desarrollar ideas innovadoras. Es importante señalar que para este autor ninguno de estos anillos aisladamente es la superdotación. Más bien, es de la interacción de estos tres conjuntos resultantes de lo que resulta esta superdotación (Renzulli, 1978). Del mismo modo, también es importante observar que cada anillo contribuye con características esenciales para que un individuo pueda tener un alto rendimiento.

En el modelo de la interdependencia triádica de Mönks (I992) se considera la superdotación como un fenómeno resultante de la interacción del individuo con el entorno. Así Mönks añade tres elementos a los anillos de Renzulli: colegio, compañeros y familia.

\section{b) Modelo diferenciado de superdotación y talento de Gagné}

El modelo diferenciado de superdotación y talento DST propone una distinción entre: superdotación y talento. En el modelo inicialmente propuesto por Gagné (1985), el término superdotación se utiliza para designar la posesión y uso de habilidades innatas superiores, no entrenadas y expresadas espontáneamente, que colocan al que las posee al menos en el percentil 90 entre sus pares de la misma edad; a su vez, en el modelo DST, el talento se refiere al dominio superior adquirido a través de una formación sistemática, que hace que el que posee ese talento tenga al menos el percentil 90 entre sus compañeros de la misma edad.

Gagné propone cuatro dominios de nivel de aptitud del nivel de superdotación: intelectual, creativo, socioafectivo y sensoriomotriz. Estas habilidades naturales pueden observarse en todas las tareas que el niño realiza a lo largo de su escolaridad.

Gagné cree que las manifestaciones de la superdotación se observan con mayor facilidad en los niños, ya que las influencias ambientales y de aprendizaje sistemáticas son moderadas. Por su parte, los talentos emergen gradualmente a medida que las habilidades superiores innatas son transformadas en experiencia en un campo en particular, a través de un entrenamiento sistemático. Por lo tanto, la existencia de habilidades superiores es un requisito previo para el acceso al talento, y esto sólo aparece cuando el niño o adolescente adquiere una práctica sistemática de aprendizaje, que Gagné denomina proceso de desarrollo. Este proceso de desarrollo está sujeto a la acción de dos tipos de catalizadores, intrapersonales y ambientales, e incluso de la aleatoriedad del factor suerte. Los catalizadores intrapersonales pueden ser de naturaleza física (por ejemplo, la salud) o mental (por ejemplo, personalidad), y el medio ambiente puede tener diversos orígenes (por ejemplo, geográfico, familiar o socioeconómico). 


\section{c) El modelo cognitivo de Sternberg}

Sternberg (1999) considera el desarrollo del talento como un proceso continuo de adquisición y consolidación de un conjunto de habilidades necesarias para un alto nivel de maestría en una o más áreas de actividad. Se refiere a las habilidades y el talento como dos caras de la misma moneda, y por lo tanto sostiene que deben ser estudiadas de una manera integrada. Así, el autor propone un modelo basado en la idea de que una persona está constantemente en proceso de desarrollo de sus habilidades cuando trabaja en un campo determinado. En este modelo subyace la idea de que el principal obstáculo en el camino hacia el talento no es un determinado nivel fijo de la capacidad anterior, sino más bien la intención de participación en la instrucción directa, la participación activa, la adaptación y la recompensa (Sternberg, 1999). Este modelo, además de admitir la influencia del contexto, incluye cinco factores nucleares que se influyen mutuamente: la capacidad metacognitiva, la capacidad de aprendizaje, la capacidad de razonamiento, el conocimiento y la motivación. Bajo esta perspectiva, el talento se adquiere a través de la práctica deliberada, pero esta práctica requiere de la interacción de los cinco elementos básicos. En el centro, guiando a estos elementos, se encuentra la motivación, un elemento clave sin el cual todos los demás son inertes. Sternberg considera que hay varios niveles de talento (por ejemplo, el del estudiante o el del profesional) y admite que el ciclo se repite de manera iterativa para niveles sucesivos de pericia.

Más recientemente, Sternberg (2005) presentó otro modelo, llamado de wics (wisdom, intelligence, creativity, synthetized), que da a la inteligencia, definida como la capacidad de adaptación al medio ambiente, el papel central. La creatividad se entiende como la capacidad de formular y resolver problemas, produciendo soluciones relativamente nuevas y de alta calidad, mientras que la sabiduría se considera como la capacidad de movilizar la inteligencia, la creatividad y el conocimiento para el bien común. De acuerdo con este diseño, la inteligencia, la sabiduría y la creatividad se alimentan mutuamente, lo que permite el desarrollo de cada uno de estos tres componentes. La idea que existe bajo este modelo es que un individuo precisa de los tres componentes trabajando simultáneamente (sintetizados) para poder contribuir favorablemente a la sociedad.

Este conjunto de modelos presentados por Sternberg hace hincapié en la idea de que el éxito se debe a varios factores, no sólo de inteligencia, que se combinan a la perfección, que convergen para formar un experto.

\section{d) Ericsson o el enfoque en la práctica deliberada}

Una línea de trabajo que difiere de los enfoques anteriores en cuanto a la visión del talento, en cuanto considera que no es algo innato al individuo, sino que hace hincapié al papel del trabajo, de la formación para la explicación del rendimiento, es la contemplada por Ericsson. Este autor forma parte de la corriente de opinión favorable a la importancia de la conducta intencional, en perjuicio de la herencia genética o talento innato.

Bajo esta perspectiva está la creencia de que no se puede acceder a altos niveles de desempeño en un área determinada sin un largo período de práctica intencional, siendo común asumir que son necesarios diez años para poder llegar a ser considerado excelente. Así, para los partidarios de este punto de vista, el criterio decisivo para el desarrollo del talento no es la presencia de factores hereditarios o los conocimientos 
¿EXISTEN INDICADORES PARA IDENTIFICAR EL TALENTO?

adquiridos en el curso de una larga experiencia, sino el compromiso con las actividades prácticas deliberadas durante al menos diez años (Ericsson et al., 1993; Ericsson et al., 1996; Ericsson et al., 1999). Por práctica deliberada se entiende una actividad estructurada, con la intención explícita de mejorar el rendimiento, la superación de dificultades, y supervisada con el fin de identificar formas de mejorar aún más (Ericsson et al., 1993).

De este modo, Ericsson y sus colaboradores se posicionan lejos de las concepciones que hacen hincapié en el papel de las habilidades innatas para explicar el alto rendimiento, y presentan un conjunto de argumentos para contrarrestar esta opinión. En particular, señalan la falta de evidencia empírica, en términos de genética, para justificar las diferencias en el rendimiento (Ericsson, Roring y Nandagopal, 2007). En el caso de los niños prodigio estos autores suponen la existencia de un ambiente fértil, que permite a estos niños estar involucrados en actividades de práctica deliberada temprana. La posibilidad que tiene una persona de involucrarse en actividades sugerentes en un momento dado puede dar lugar a pequeñas ventajas que tengan una importancia decisiva en su desempeño futuro, y que en realidad le conduzcan a ser mejor que sus pares en el futuro (Gladwell, 2008).

En resumen, ha habido una gran diversidad de tentativas y posicionamientos en cuanto a la explicación del talento desde aquellos modelos que reducen dicha explicación a los factores innatos, aquellos que consideran la influencia de factores ambientales y hasta aquellos que ponen su énfasis en la instrucción y una práctica deliberada continuada.

\section{Algunos elementos que caracterizan el talento}

Entre los indicadores que hemos visto que los investigadores relacionan con el talento existe una gran diversidad. A continuación destacaremos los que consideramos que actualmente ponen más de relieve las características del talento. Entre ellos se encuentran: la motivación, la autorregulación, la creatividad o el pensamiento divergente.

Un individuo dotado de una gran capacidad intelectual suele tener altos niveles de motivación, con lo que logra buenos niveles de rendimiento, pero puede mejorar su rendimiento si utiliza en la medida de lo posible estrategias de autorregulación.

Con estas estrategias la persona decide el camino para llevar a cabo una tarea, cualquiera que sea la naturaleza de esta misión. Ejemplos de técnicas de autorregulación son: el establecimiento de objetivos (especificación de acciones o resultados de rendimiento); la elección de las estrategias para realizar una tarea específica; la autoinstrucción (expresiones explícitas o veladas para mejorar el rendimiento, por ejemplo, las frases que conducen a la autorrelajación para reducir la ansiedad); la creación de imágenes mentales (secuencias de comportamiento, para mejorar el rendimiento); la gestión del tiempo; el automonitoreo (seguimiento del propio rendimiento); la autoevaluación (se refiere a la fijación y el uso de los niveles de calidad realistas para medir el progreso); la estructuración del ambiente (se refiere a la selección y creación de un lugar adecuado para realizar la tarea); y la búsqueda de ayuda (se refiere a la elección de las fuentes de conocimiento o habilidades, por ejemplo, los maestros, los libros o los modelos) (Zimmerman, 2002). 
En el campo educativo, un estudiante autorregulado ve la adquisición de conocimiento como un proceso controlable, lo que le lleva a aceptar una mayor responsabilidad por los resultados obtenidos que sus compañeros. Un estudiante activo con su aprendizaje implementa esta regulación a través de un enfoque diligente al nivel motivacional, comportamental y metacognitivo (Zimmerman, 1990). Acerca de los procesos de motivación, los estudiantes autorregulados muestran elevados índices de autoeficacia y de motivación intrínseca en la tarea, están dispuestos esforzarse y a persistir en el aprendizaje. En cuanto a los procesos de comportamiento del estudiante autorregulado, se caracterizan por la elección y la creación de entornos que le permiten optimizar su aprendizaje. Entre los procesos metacognitivos distintivos de un estudiante autorregulado están la planificación, el establecimiento de objetivos, la organización, las actividades de seguimiento y la autoevaluación en diversos momentos a lo largo del proceso de adquisición de conocimientos (Zimmerman, 1990).

Otro elemento que está relacionado con el talento es la motivación. El término motivación (procedente del latín, que significa mover) se refiere a los factores, sean necesidades o deseos, que impulsan un comportamiento propicio para un propósito particular.

Hay un conjunto de teorías sobre la motivación que tratan de explicar cómo un individuo se inicia y mantiene su motivación, es decir, cómo es la motivación. Un ejemplo de este enfoque es el considerado por Adams (1963), conocido por teoría de la equidad, que sugiere que si un individuo percibe que las recompensas son recibidas de forma equitativa cuando se compara con otro, entonces el individuo siente satisfacción. En este sentido, una persona trata de que haya un equilibrio entre lo que trabaja y los resultados obtenidos, y si siente que este no es el caso y que se encuentra en desventaja tiende a adaptar su comportamiento para reducir esta discrepancia.

Otro ejemplo es la teoría desarrollada por Vroom (1964), que supone que la motivación de un individuo a actuar de cierta manera está determinada por su expectativa de que su conducta traerá un resultado en particular. En esta perspectiva, la motivación del individuo resulta del producto, de la expectativa (su percepción de que sus esfuerzos darán como resultado un rendimiento dado) a través de la instrumentalidad (su percepción de que su desempeño será recompensado) y valencia (su percepción del valor de la recompensa que será el resultado de su actuación).

Más recientemente, Deci y Ryan (1985) presentan la teoría de la autodeterminación, donde, de forma integral, combinan la motivación humana y la personalidad. Inherente a este enfoque está la suposición de que hay tres necesidades psicológicas universales e innatas (competencia, autonomía e interacción) que motivan a los individuos en su búsqueda de la salud y el bienestar.

Así, se han propuesto numerosas teorías con la intención de explicar la motivación y aunque cada una de ellas ha aportado parte de lo que conocemos actualmente acerca de este tema, parece que ninguna, por sí misma, explica adecuadamente la motivación humana (Williams y Williams, 20II).

La motivación de un individuo puede originarse en sí mismo, por lo que tendría un origen intrínseco, o puede ser el resultado de causas externas, conocidas como la motivación extrínseca.

Se trata de la motivación intrínseca cuando la actividad en cuestión se lleva a cabo debido a la satisfacción que da, el desafío, su interés o para satisfacer una curiosidad. En este caso, una persona se mueve a actuar para la diversión o desafío que implica 
una tarea, en lugar de hacerlo debido a factores externos. Estas acciones no parecen estar causadas por una razón instrumental, sino por las experiencias positivas asociadas con el ejercicio (Ryan y Deci, 200o), se está actuando de acuerdo con los propios intereses de un individuo por su necesidad de conocimiento y para mejorar sus habilidades. Amabile (1983) considera la existencia de la motivación intrínseca como requisito previo para la realización de un trabajo creativo.

A pesar de que la motivación intrínseca es un tipo importante de motivación, la mayoría de las actividades que las personas realizan lo hacen no por estar intrínsecamente motivadas. La motivación extrínseca contrasta con la motivación intrínseca en el sentido de que la actividad se lleva a cabo a causa de factores externos al individuo (Deci y Ryan, 200o). En este sentido un individuo puede ser extrínsecamente motivado en diversos grados. Por ejemplo, un estudiante que decide hacer su tarea para evitar sanciones de los padres está motivado extrínsecamente, ya que lo hace para evitar el castigo. Por otra parte, un estudiante que hace su tarea porque cree que es importante para su futura profesión también está motivado extrínsecamente, ya que no lo hace para obtener satisfacción inherente, sino más bien por las consecuencias externas. En ambos casos, está el valor instrumental del trabajo realizado en casa, pero mientras que en el segundo la aprobación personal del estudiante es en el sentido de la elección, en la primera sólo hay una sujeción a un control externo.

Otro indicador interesante en cuanto a la caracterización del talento es la creatividad. Las definiciones de creatividad difieren, pero tienen en común el énfasis en la capacidad del individuo para hacer productos que no sólo sean de buena calidad, sino que también sean originales (Sternberg, 200I). Aunque existen publicaciones científicas sobre el tema de la creatividad antes de 1950, el inicio de la investigación sobre creatividad se asigna a Guilford (1950), que en un discurso ante la Asociación Americana de Psicología (APA) advirtió de la importancia de la creatividad y la clasificó como un recurso natural.

Paul Torrance (1988) define el pensamiento creativo como el proceso de llegar a ser sensibles a los problemas, las lagunas de información, falta de elementos, desarmonías; hacer suposiciones y formular hipótesis acerca de estas imperfecciones; evaluar y probar estos supuestos e hipótesis; posiblemente modificarlos, perfeccionándolos y volver a probarlos; $y$, finalmente, informar de los resultados. El mismo autor entiende la creatividad como un proceso natural en el que las necesidades humanas están en la base de todos los procesos creativos, una manera de responder constructivamente a las situaciones, en lugar de simplemente adaptarse a ellas; así que la creatividad es un atributo observable de la realidad cotidiana y no sólo de los altos niveles de la creación (Torrance, 1993). Describiendo la creatividad, Torrance (1992) señala algunas características personales asociadas, tales como la tolerancia a fallos, el placer de la propia obra, el valor para asumir ideas creativas, el gusto por el desafío o la persistencia.

En cuanto al pensamiento divergente, la concepción actual se basa en el concepto de producción divergente de Guilford. El pensamiento divergente se entiende como un acercamiento a una situación o concepto como la exploración de tantos aspectos como sea posible. Comenzando con una sola idea, una persona con pensamiento divergente permite que su mente se distraiga en diferentes direcciones, añadiendo numerosos pensamientos relacionados con el tema en cuestión, lo que puede dar lugar a diferentes respuestas a un solo problema. Esto difiere del pensamiento convergente, a menudo asociado con la inteligencia, en el que varias ideas se unen para diseñar una 
única respuesta, por lo general después de una secuencia de pasos lógicos para llegar a ese logro.

El pensamiento divergente se define como la capacidad cognitiva para producir variadas y numerosas ideas para una tarea o problema (Runco, I991; Amabile, 1996). La capacidad de pensamiento divergente es considerada como uno de los factores cognitivos más importantes que pueden conducir a la creatividad (Amabile, i996; Plucker y Renzulli, 1999; Plucker et al., 2006) y las pruebas de pensamiento divergente son probablemente la forma de medir la creatividad más utilizada (por ejemplo, Runco, 1993).

\section{Sobre este monográfico}

Este monográfico pretende dar a la comunidad educativa algunas de las ideas y de los resultados de investigaciones en curso que se están realizando sobre el talento, así como experiencias para profesores y futuros profesores para que integren en sus aulas a los alumnos con talento tal como indicábamos al inicio de este artículo.

El monográfico está integrado por cinco artículos además de este en el que se ha tratado de identificar el talento, dos de índole trasversal en el que el talento se trata de forma general sin hacer referencia a un talento en particular y tres de ellos centrados en algún talento especial: el talento musical, el talento matemático y el talento en el deporte.

El artículo «Cómo funcionan las competencias socioemocionales en los estudiantes de alta habilidad» muestra un estudio cuantitativo con estudiantes de educación secundaria obligatoria en el que se analizan las competencias socioemocionales de alumnos de II a I7 años, entre los que se encuentran algunos alumnos con diferentes tipos de talento o diferentes competencias. En esta investigación se han centrado en los estudiantes con talento académico, figurativo o combinado entre ambos.

En el siguiente artículo, "Creatividad científica y alta habilidad: diferencias de género y nivel educativo", que está centrado en uno de los indicadores que hemos establecido en este artículo que caracterizan el talento que es la creatividad, se han analizado, a través de un test, la creatividad general, las habilidades científicas y el conocimiento que tienen los alumnos de II a I4 años en las áreas de ciencias.

Como se ha indicado anteriormente los siguientes artículos están enfocados a algún talento en particular. A pesar de que cada uno de ellos aborda un talento diferente todos ellos muestran un elemento común y es la idea de romper con las concepciones y las formas de hacer tradicionales.

El primero de ellos está dedicado al talento musical y en él se hace una reflexión interesante sobre la formación musical. Se plantea la idea de que la enseñanza de la música siempre ha pivotado en torno al talento y las modernas concepciones están más bien enfocadas en torno a la inteligencia musical, que implica una enseñanza más flexible, integradora y abarcadora de metodologías innovadoras distintas de las meramente tradicionales.

En cuanto al talento matemático se plantea un reto para los profesores respecto a la atención a los alumnos que lo poseen. Una forma de abordar esta situación es la de crear en el aula situaciones que supongan un reto para los alumnos como fuente de aprendizaje de las matemáticas de forma que superen el aburrimiento que les suponen las tareas rutinarias que se proponen en las aulas. 
Finalmente, en el último artículo se aborda el talento en el deporte. Así se problematiza la detección del talento deportivo cuando se considera una capacidad innata al individuo en los mismos términos en los que se ha indicado en el presente este artículo. Si se considera el talento como un concepto multidimensional y dinámico que varía con el tiempo, el tipo de mecanismos que se deben usar para detectar el talento también ha de adaptarse a esta nueva concepción.

Todas estas investigaciones y propuestas de enseñanza y de aplicación en relación con el talento dan una visión panorámica de lo que se considera el talento así como una visión local de algunos talentos particulares que son interesantes para la comunidad educativa en general y para los futuros profesores en particular por la ayuda que les pueda ofrecer en su labor cotidiana.

\section{Bibliografía}

Adams, J. S. (1963) Towards an understanding of inequity. Journal of Abnormal and Social Psychology, 67, 422-436.

http://dx.doi.org/Io.I037/hoo40968

Amabile, T. M. (1983) The Social Psychology of Creativity. New York: Springer-Verlag. http://dx.doi.org/I0.1007/978-I-46I2-5533-8

Амabile, T. M. (1996) Creativity in context: Update to the social psychology of creativity. Boulder: Westview Press.

Castelló, A. y Battle, C. (1998) Aspectos teóricos e instrumentales en la identificación del alumno superdotado y talentoso. Propuesta de un protocolo. FAISCA, 6, 26-66. Santiago de Compostela.

CHI, M. T. H. (2006) Laboratory methods for assessing experts' and novices' knowledge. En K. Anders Ericsson, Neil Charness, Robert R. Hoffman y Paul J. Feltovich (eds.) The Cambridge handbook of expertise and expert performance (pp. 167-184). Cambridge: Cambridge University Press. http://dx.doi.org/Io.IoI7/CBO9780511816796.oIo

Cianciolo, A. T.; Matthew, C.; Sternberg, R. J. y Wagner, R. K. (2006) Tacit knowledge, practical intelligence, and expertise. En K. Anders Ericsson, Neil Charness, Robert R. Hoffman y Paul J. Feltovich (eds.) The Cambridge handbook of expertise and expert performance (pp. 613-632). Cambridge: Cambridge University Press. http://dx.doi.org/ro.Ior7/CBO9780511816796.035

Deci, E. L. y Ryan, R. M. (1985) Intrinsic motivation and self-determination in buman behaviour. New York: Plenum. http://dx.doi.org/Io.1007/978-I-4899-227I-7

ERICSSON, K. A. (2009) Enhancing the development of professional performance: implications from the study of deliberate practice. En K. A. ErICsSON (ed.) Development of professional expertise toward measurement of expert performance and design of optimal learning environments (pp. 405-431). Cambridge: Cambridge University Press.

http://dx.doi.org/Io.1017/CBO9780511609817

http://dx.doi.org/ıo.1017/CBO9780511609817.022

Ericsson, K. A.; Krampe, R. T. y Tesch-Römer, C. (1993) The role of deliberate practice in the acquisition of expert performance. Psychological Review, 100, 363-406. http://dx.doi.org/IO.I037/0033-295X.100.3.363

Ericsson, K. A. y Lehmann, A. C. (1996) Expert and exceptional performance: Evidence of maximal adaptation to task constraints. Annual Review of Psychology, 47, 273-305. http://dx.doi.org/Io.II46/annurev.psych.47.I.273 
Ericsson, K. A. y Lehmann, A. C. (1999) Expertise. En M. A. Runco y S. R. Pritzker (eds.) Encyclopedia of Creativity, vol. I (pp. 695-707). San Diego: Academic Press.

Ericsson, K. A.; Roring, R. W. y Nandagopal, K. (2007) Giftedness and evidence for reproducibly superior performance: An account based on the expert performance framework. High Ability Studies, I8 (I), 3-56.

http://dx.doi.org/10.1080/13598130701350593

GAGNÉ, F. (1985) Giftedness and talent: Reexamining a reexamination of the definitions. Gifted Child Quarterly, 29 (3), I03-112. http://dx.doi.org/Io.II77/001698628502900302

Gladwell, M. (2008) Outliers. New York: Little, Brown and Company.

Guilford, J. P. (1950) Creativity. American Psychologist, 5 (9), 444-454. http://dx.doi.org/I0.I037/hoo63487

Martínez, M. y Guirado, A. (coords.); Prieto, A.; Reverter, R.; Ruiz, N. y Valera, M. (2012) Altas capacidades intelectuales. Pautas de actuación, orientación, intervención y evaluación en el periodo escolar. Barcelona: Editorial Graó.

Plucker, J. A. y Renzulli, J. S. (1999) Psychometric approaches to the study of human creativity. En R. J. Sternberg (ed.) Handbook of creativity (pp. 35-6I). Cambridge: Cambridge University Press.

Plucker, J. A.; Runco, M. A. y Lim, W. (2006) Predicting ideational behavior from divergent thinking and discretionary time on task. Creativity Research Journal, I8 (I), 55-63. http://dx.doi.org/IO.I207/sI5326934crji8oI_7

Prieto, M. D. y CASTejón, J. L. (2000) Los superdotados: esos alumnos excepcionales. Málaga: Ediciones Aljibe S. L.

Renzulli, J. S. (1978) What makes giftedness? Reexamining a definition. Recuperado el in de marzo de 20I4, de http://www.mishawaka.kı2.in.us/documents/HA\% 2odocs/EDPS\% 20 540\% 20articles/Module\% 201\% 20-\% 202\% 20(January\% 2026)/Renzulli.pdf.

Runco, M. A. (1991) Divergent thinking. Norwood: Ablex Publishing Corporation.

Runco, M. A. (I993) Divergent thinking, creativity, and giftedness. Gifted Child Quarterly, $37,16-22$.

http://dx.doi.org/ıo.1177/0o1698629303700103

Ryan, R. M. y Deci, E. L. (200o) Intrinsic and extrinsic motivations: Classic definitions and new directions. Contemporary Educational Psychology, 25 (I), 54-67.

http://dx.doi.org/Io.Ioo6/ceps.1999.1020

Sternberg, R. J. (1999) Intelligence as developing expertise. Contemporary Educational Psychology, 24, 359-375. http://dx.doi.org/IO.IOo6/ceps.1998.0998

Sternberg, R. J. (200I) What is the common thread of creativity? Its dialectical relation to intelligence and wisdom. American Psychologist, 56 (4), 360-362.

http://dx.doi.org/10.1037/0003-066X.56.4.360

Sternberg, R. J. (2005) wiCs: A model of leadership. The Psychologist Manager Journal, 8 (I), 29-43.

http://dx.doi.org/Io.I207/sI550346Itpmjo8oI_4

Torrance, E. P. (1988) The nature of creativity as manifest in its testing. En R. J. STernberg (ed.) The nature of creativity: Contemporary psychological perspectives (pp. 43-75). Cambridge: Cambridge University.

Torrance, E. P. (1992) The beyonders in a thirty year longitudinal study of creative achievement. Roeper Review, I5 (3), I3I-I35.

Torrance, E. P. (1993) Understanding creativity: Where to start? Psychological Inquiry, 4 (3), 232-234. http://dx.doi.org/IO.I207/si5327965plio403_I7

VanLehn, K. y Van De Sande, B. (2009) Acquiring conceptual expertise from modeling: the case of elementary physics. En K. A. ERICsson (ed.) Development of professional expertise 
toward measurement of expert performance and design of optimal learning environments (pp. 356-378). Cambridge: Cambridge University Press.

http://dx.doi.org/Io.Ior7/CBO9780511609817.020

Vroom, V. H. (1964) Work and Motivation. New York: John Wiley \& Sons.

Williams, K. C. y Williams, C. C. (20II) Five key ingredients for improving student motivation. Research in Higher Education Journal, I2, I04-122.

Zimmerman, B. J. (1990). Self-regulated learning and academic achievement: an overview. Educational Psychologist, 25 (I), 3-I7. http://dx.doi.org/Io.1017/CBO9780511609817.020

Zimmerman, B. J. (2002) Achieving academic excellence: A self-regulatory perspective. En Michel Ferrari (ed.) The Pursuit of Excellence Through Education (pp. 85-III). Mahwah: Lawrence Erlbaum Associates, Inc.

Zimmerman, B. J. (2006) Development and adaptation of expertise: The role of self-regulatory processes and beliefs. En K. Anders Ericsson, Neil Charness, Robert R. Hoffman y Paul J. Feltovich (eds.) The Cambridge handbook of expertise and expert performance (pp. 705 722). Cambridge: Cambridge University Press.

http://dx.doi.org/Io.10I7/CBO9780511816796.039 